\title{
Los colores subliminales en la obra de José Guerrero. Metodología y análisis para el deleite
}

\section{Subliminal colours in the Jose Guerrero's work. Methodology and analysis for the pleasure}

\author{
María del Carmen Bellido \\ Universidad de Granada. Facultad de Bellas Artes. Departamento de Escultura \\ cbellido@ugr.es
}

Recibido: 17 de septiembre de 2012

Aprobado: 23 de marzo de 2013

\section{Resumen}

La colección del Centro José Guerrero de Granada cuenta con un considerable número de pinturas de este autor, que suponen una muestra importante de su producción artística. En los últimos años, gracias al entendimiento y la colaboración entre diferentes organismos públicos, se ha terminado con la polémica sobre la permanencia de esta colección en el museo. Una extensa investigación realizada sobre las obras, mediante el estudio colorimétrico de sus superficies pictóricas, ha permitido conocer novedosos aspectos artísticos de ellas, como degradados en aparentes manchas de colores planos, límites lineales o difuminados entre diferentes zonas y, especialmente, áreas de coloridos casi inapreciables mediante análisis visual al natural, aspectos que han quedado demostrados mediante metodología científica.

El trabajo también ha contribuido a conocer mejor las técnicas creativas y los colores utilizados, junto a la expresividad de las obras, aspectos que han verificado su calidad. Sus gamas de colores son estímulos visuales que para muchos observadores resultan inapreciables conscientemente, debido a las capacidades de diferenciación del color que poseen, pero que pueden influir en las reacciones que experimentan ante su contemplación, a causa de la apreciación inconsciente. La percepción subliminal de los colores no visibles aparentemente y la sinestesia justifican este hecho.

Palabras clave: arte, conservación, pintura, colorimetría, sinestesia.

Bellido, M.C. (2013): Los colores subliminales en la obra de José Guerrero. Metodología y análisis para el deleite. Arte, Individuo y Sociedad, 25(3) 441-457

\begin{abstract}
José Guerrero' s Art Centre collection shows a considerable number of paintings by this author, which are a great part of his artistic work. In the last years, thanks to the understanding and collaboration among different public entities, the controversy regarding the permanence of the collection in the museum has ended. A wide research has been done about the paintings through the colorimetrical study of its pictorial surfaces, which shows new artistic aspects of them. Some of these are: graduated shading in apparent stains of plain colours, linear limits or shades among different parts and, specially, areas with almost negligible colour through natural visual analysis. These have been shown thanks to the scientific methodology used.

The research has also contributed to learn about the creative techniques, the colours and the expressivity of his paintings, verifying their quality. Their range of colours is visual stimulus that for many observers may seem consciously imperceptible due to their capacity of differenciating the colours shown. However, they may have an influence on the reactions the observers might experience when they contemplate the paintings because of the unconscious appreciation. The subliminal apparently appreciated and the synesthesia justify this phenomenon.
\end{abstract}

Key words: art, conservation, painting, colorimetry, synesthesia. 
Bellido, M.C. (2013): Subliminal colours in the Jose Guerrero's work. Methodology and analysis for the pleasure. Arte, Individuo y Sociedad, 25(3) 441-457

Sumario: 1. Introducción y objetivos, 1.1. Contextualización social, 1.2. Objetivos, 2. Metodología, 2.1. Materiales, 2.2. Equipo instrumental, 2.3. Elementos auxiliares, 3. Resultados y discusión, 3.1. Solitarios (1972), 3.2. Solitarios (1971), 3.3. Intervalos negros (1971), 4. Conclusiones. Referencias.

Este artículo recoge resultados de la investigación de la Tesis Doctoral titulada Estudio conservacional y análisis material de obras de arte contemporáneo. Un caso experimental: colección del Centro José Guerrero (Bellido, 2010), que ha sido financiada con una Beca de Formación de Profesor Universitario (2006-2010) del Ministerio de Educación y Ciencia y una Beca del Plan Propio de la Universidad de Granada (20052006). También ha contado con la financiación del Proyecto de Investigación MAT 2006-00308 (Ministerio de Educación y Ciencia) y la Acción Integrada HP2008-0037, concedidos al Grupo de Investigación HUM-629 de la Junta de Andalucía (Universidad de Granada).

\section{Introducción y objetivos}

\subsection{Contextualización social}

El Centro José Guerrero está localizado en la calle Oficios, $n^{\circ} 8$ de Granada (Centro José Guerrero, 2012) y fue inaugurado en el año 2000, contando con una colección permanente del pintor José Guerrero (Granada, 1914 - Barcelona, 1991). Las obras de esta colección pertenecen a la familia Guerrero y fueron cedidas a la Diputación de Granada. En los últimos años hubo desacuerdos de gestión sobre la continuidad de las mismas en el museo, durante los que se temió por la dificultad que esto supondría para concluir las investigaciones iniciadas en este estudio y por la pérdida cultural que sufriría la ciudad de Granada.

Las discrepancias (2009-2011) entre la Diputación de Granada, propietaria y gestora del Centro José Guerrero y la familia Guerrero (formada por los herederos del artista), han sido los motivos por los que se temió por el traslado fuera de Andalucía de las obras pictóricas de este autor, que alberga dicha institución artística desde su fundación y apertura (2000). Se trata de una colección de arte contemporáneo muy relevante, formada por sesenta obras (pictóricas y gráficas), las cuales son una muestra de toda la trayectoria artística del pintor, teniendo especial relevancia un buen número de ellas realizadas entre 1950-1970 en Nueva York. A pesar de las dificultades y los temores citados, desde 2006, en que se inició esta investigación, se ha conseguido realizar un estudio relevante sobre las condiciones expositivas y colorimétricas de los cuadros, que ha puesto de manifiesto las características del color y las técnicas empleadas por el artista (Bellido, Durán y Peralbo, 2008). Esto permite una nueva apreciación de las pinturas, 
gracias a la definición científica de la percepción visual de las mismas, lo que ayuda a interpretar las posibles causas de las emociones que pueden llegar a experimentar los espectadores frente a una de estas obras, a partir del conocimiento de como están realmente coloreadas.

El Centro J. Guerrero se creó por iniciativa de la Diputación de Granada, dada la importancia que tiene la producción artística de José Guerrero en el contexto del arte de la segunda mitad del siglo XX, que está directamente relacionada con el Expresionismo Abstracto norteamericano y la Escuela de Nueva York, junto a la influencia que tuvo esta obra en la creación plástica española durante la transición democrática, además de la influencia que ha ejercido después en artistas más contemporáneos y actuales.

La colección está compuesta por un legado importante, formado por cuarenta obras al óleo sobre lienzo (Guerrero, 2000) y veinte hechas con técnicas mixtas sobre papel, además de un valioso archivo documental personal del artista y su biblioteca de arte (Centro José Guerrero, 2012). El legado completo está valorado en casi 10.000.000 € (Arias, 2009). Las obras pictóricas forman una relevante muestra de toda su trayectoria creativa, según se puede ver en los libros José Guerrero: Catálogo Razonado, volumen I y II, que comprenden todos sus trabajos conocidos (Romero y Vallejo, 2007, 2008).

El acuerdo de cesión de esta colección fue formalizado en el año 2000, con un contrato de comodato, quedando la familia Guerrero como propietaria de las obras, que fueron cedidas a la Diputación de Granada (propietaria del Centro Guerrero) por un periodo de diez años, pasados los cuales ambas partes acordarían su cesión definitiva a la provincia de Granada, si el funcionamiento del Centro había resultado satisfactorio.

En opinión general, la actividad del Centro Guerrero ha sido valorada siempre como positiva. Su labor ha sido encomiable, habiendo contribuido a la permanencia de la memoria del artista y habiéndose definido como un espacio público que ha acogido la vanguardia cultural (Millán, 2009). Además, ha mantenido la colección en buen estado de conservación (Bellido y Durán, 2007), ha permitido la investigación y ha conseguido ser uno de los lugares más visitados de Granada.

Sin embargo, las desavenencias entre los representantes de la Diputación y la familia Guerrero comenzaron a hacerse públicas a principios de 2009, cuando el Centro de arte preparaba la programación de las actividades culturales de 2010, dedicadas a celebrar la primera década de su apertura, en un ambiente dicotómico (Fig. 1). Por un lado, estaba la satisfacción por el trabajo bien hecho y por otro, la discordia surgida entre ambas partes, debido a divergencias de opiniones e intereses. El contrato de cesión de las obras terminaba a final de 2010, por lo que se acercaba el momento de reformar el acuerdo inicial o darlo por terminado y retirar la colección de Granada.

Pero la polémica estaba abierta. Venía surgiendo desde el año anterior, cuando la Diputación de Granada propuso la creación de una Fundación Granadina de Arte Contemporáneo, en la que la colección del Centro José Guerrero quedaría incluida junto a otros fondos artísticos de la institución provincial. La familia Guerrero rechazaba esta idea, porque entendía que esto debilitaría la identidad del Centro, de la colección y de la memoria de la obra de su padre, y apuntó la posibilidad de trasladar el legado. Pero también afirmó que la única forma de mantener la cesión de las obras pasaba por la creación de una fundación, en la que intervinieran otras instituciones y que fuera gestionada por el Centro J. Guerrero. Se quería negociar la participación en ella de la 
Junta de Andalucía, el Ayuntamiento de Granada y el Ministerio (Gallastegui, 2010). La oferta de los herederos de Guerrero ofrecía la voluntad de mantener el diálogo entre ambas partes y aceptar una prórroga del convenio de cesión de la colección, mientras se solventaba el problema.
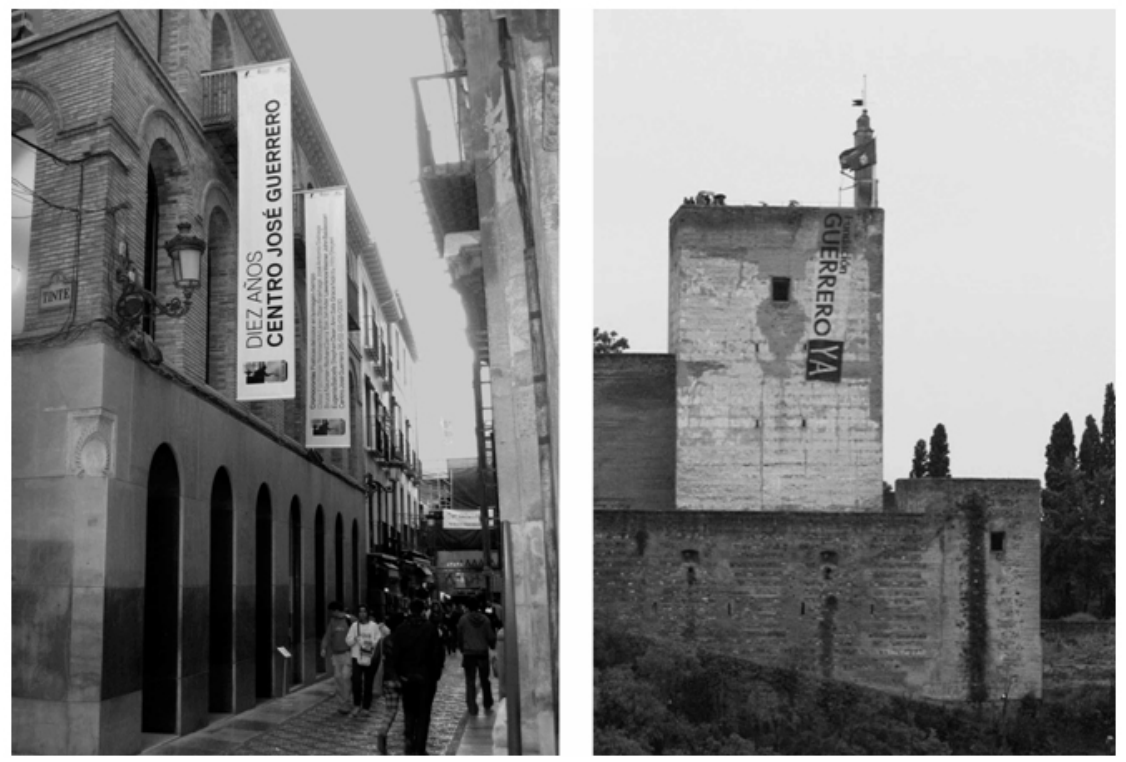

Figura 1A. Fachada del Centro José Guerrero con una cartela conmemorativa de sus diez años de apertura (2010), autoría: M. C. Bellido Márquez. Figura 1B. Muros la Torre de la Vela del monumento histórico de la Alhambra con una pancarta de apoyo al Centro José Guerrero. Imagen tomada del vídeo Pancarta por el Centro Guerrero en la Torre de la Vela, procedencia: idealtv.es, 2009.

Paralelamente, fue destacable la respuesta de la opinión pública en este conflicto, pues un importante colectivo de personas, vinculadas al arte y la cultura, crearon la plataforma Por el Centro José Guerrero (Por el Centro J. Guerrero, 2010), la cual protagonizó movilizaciones de protesta (Arias, 2009) (Fig. 1B). Con tales reacciones, la Diputación abandonó sus propósitos iniciales y en junio de 2009 propuso la apertura del Centro de Creación Artística José Guerrero, con el compromiso de iniciar los contactos para que el Gobierno Central y la Junta de Andalucía formaran parte de una Fundación que llevara el nombre de José Guerrero.

Durante el transcurso de 2010 se mantuvieron las discrepancias entre la Diputación de Granada y la familia Guerrero. El 18 de septiembre de ese año se publicó en el periódico El Ideal el nuevo desacuerdo entre las partes (Gallastegui, 2010). Por último, surgieron entendimientos y se acordó por consenso crear la Fundación José Guerrero de Arte Contemporáneo, con participación de Ayuntamiento de Granada, la Junta de Andalucía, el Ministerio de Educación y Cultura, además de la Diputación de Granada. También, se prorrogó el contrato de cesión, hasta que se hubiera concretado definitivamente el modelo de gestión a seguir (Europa Press, 2011).

Entre todas estas vicisitudes y temiendo que en cualquier momento la colección 
Guerrero fuera retirada de Granada, dejando parte de este trabajo de investigación (iniciado en 2006) sin concluir, se continuó con él sin dilación, con gran constancia, esfuerzo y optimismo hasta obtener resultados (Bellido, 2010). Es de agradecer que en ningún momento hubo impedimento alguno para que se hiciera este estudio, que siempre fue considerado como muy conveniente para optimizar la conservación de las obras pictóricas y novedoso para su evaluación colorimétrica, dado que supone la aportación de nuevos análisis basados en técnicas científicas, que permiten mejorar su valoración artística y la compresión de su disfrute perceptual.

Para iniciar el trabajo, se ha partido del estudio de la técnica artística del pintor y del uso que hizo del color. Para Guerrero la selección de los colores tenía una especial importancia. Cada uno de ellos poseía un significado personal, atribuido a sus recuerdos y vivencias. En sus obras, los colores expresan simbologías y significados referidos a paisajes vivenciales, formando un abanico de percepciones visuales que ahondan en los sentimientos y en el imaginario sensorial de quien las contempla. El artista relacionaba el añil con los zócalos de su tierra andaluza y las muñequillas de azulillo que hacía su madre para teñir la ropa. El azul celeste hacía referencia a su infancia. El rojo era la almagra obtenida de la tierra en estado natural (óxido de hierro), usado como colorante en pinturas. Así se expresó el pintor sobre estos dos últimos colores: "casi son los dos colores más importantes para mí, el rojo y el azul” (Ortuño, 1981, p. 147). El negro era para él la tragedia, el color de los colores, usado mucho en su época más expresionista. El amarillo y el verde representaban el campo y la vegetación, recuerdo de sus paseos campestres. El blanco era la propia Andalucía, sus casas y luminosidad. Los malvas y violetas los asociaba a las frutas (el higo chumbo, la breva, la ciruela). El ocre era la tierra sobre la que jugó. Los grises eran los montes de Andalucía, especialmente los de Sierra Nevada en Granada (Ortuño, 1981).

\subsection{Objetivos}

El objetivo principal del trabajo ha sido aportar información de manera científica a las posibles causas de las sensaciones perceptuales que experimenta el observador ante las coloridas obras de José Guerrero, mediante una metodología que permite definir en ellas los campos de color, los límites (lineales o difusos) entre planos o la definición científica de sus colores, entre otras características, y conocer con exactitud los parámetros de color que las forman, incluso detectando aquellos de carácter subliminal y creando un registro de datos que ayude a mejorar la conservación preventiva de las obras. Los objetivos siguientes son complementarios al anterior y se han planteado para poder realizar la actividad propuesta.

En primer lugar, implementar los conocimientos sobre el uso de las técnicas con las que José Guerrero realizó las obras, las aplicaciones matéricas, las combinaciones de color y las mixturas visuales utilizadas. Esto ampliará la información con que se cuenta actualmente, que consiste en análisis visuales y químicos que determinan que las obras pictóricas están hechas principalmente al óleo sobre lienzo, sobre bases de imprimación blanca.

Seguidamente, conocer las características colorimétricas de las obras de la colección del Centro seleccionadas para el estudio, mediante la realización de ensayos de color 
cuyos resultados queden expresados en gráficos que permitan el estudio de las mismas. Para obtener las características numéricas de los colores se usará el sistema internacional de mediciones colorimétricas CIELAB 1976, que está aprobado por la Commission Internationale de lÉclairage CIE, responsable de la coordinación internacional de las normas técnicas de medición de la iluminación. La estandarización de este sistema permitirá que los datos sean legibles e interpretados por diferentes medios con los mismos resultados, evitando la percepción subjetiva del color y, por el contrario, objetivando los resultados.

Por último, elaborar un registro de los datos obtenidos según mediciones normalizadas del color, que permita comprobar a medio y largo plazo la evolución colorimétrica que puedan sufrir las pinturas, incluso, antes de que la percepción visual humana las detecte. Pues gracias al actual registro numérico de los colores, se podrá llevar a cabo una investigación continuada sobre las variaciones que se puedan producir en el futuro, al advertir las posibles diferencias entre los datos de este estudio y los obtenidos con posterioridad. Los cambios de color, comprobados mediante la variación de las cifras, podrán ser detectados incluso antes de que la percepción humana los aprecie y se podrán conocer los parámetros de color que han sufrido variaciones, así como la tendencia al cambio que experimenten las superficies pictóricas; ante esto, lo más adecuado será estudiar las causas de dichos cambios, para procurar evitarlos, minimizarlos o ralentizarlos. A partir de aquí, se tendrá una información que orientará adecuadamente a definir los criterios que fijen las medidas de conservación preventiva para las pinturas, que eviten, en lo posible, que las modificaciones del color se agraven y afecten a la contemplación de las obras.

\section{Metodología}

\subsection{Materiales}

La investigación se ha realizado sobre once cuadros originales de José Guerrero, pertenecientes a la colección Guerrero de Granada, pero dada la amplitud de todo el trabajo, en esta publicación se presenta el estudio de sólo tres de ellos (Figura 2), como ejemplos que muestran las conclusiones de la investigación: Solitarios (1972), Solitarios (1971), Intervalos negros (1971). Son obras pertenecientes a la serie denominada Fosforescencias (Guerrero, 2003).

\subsection{Equipo instrumental}

Las mediciones colorimétricas se realizaron con un espectrofotómetro digital, equipo de análisis del color que registra y almacena datos obtenidos mediante ensayos, se complementa con un software y permite ejecutar funciones concretas con los registros obtenidos. Con esta técnica se obtienen análisis de colorimetría, que definen las magnitudes del espectro visible. Además, ésta supone un procedimiento adecuado para conseguir el propósito de expresar los atributos perceptuales del color de manera objetiva, mediante datos numéricos.

El color se midió con un Espectrofotómetro de Reflectancia CM-2500c Konica 
Minolta. Se trata de un espectrofotómetro portátil de mano, con geometría 45/0, para medición y control del color, iluminación en forma de anillo a $45^{\circ}$, observación vertical, donde la muestra se ilumina desde un ángulo de $45^{\circ}$, con respecto a la superficie, y la luz reflejada se mide desde un ángulo $0^{\circ}$. Los parámetros de color que utiliza este equipo están basados en el sistema CIELAB 1976, espacio de color normalizado derivado del anterior CIE 1931, fundamentado en el triestímulo óptico XYZ (rojo, verde, azul, respectivamente).
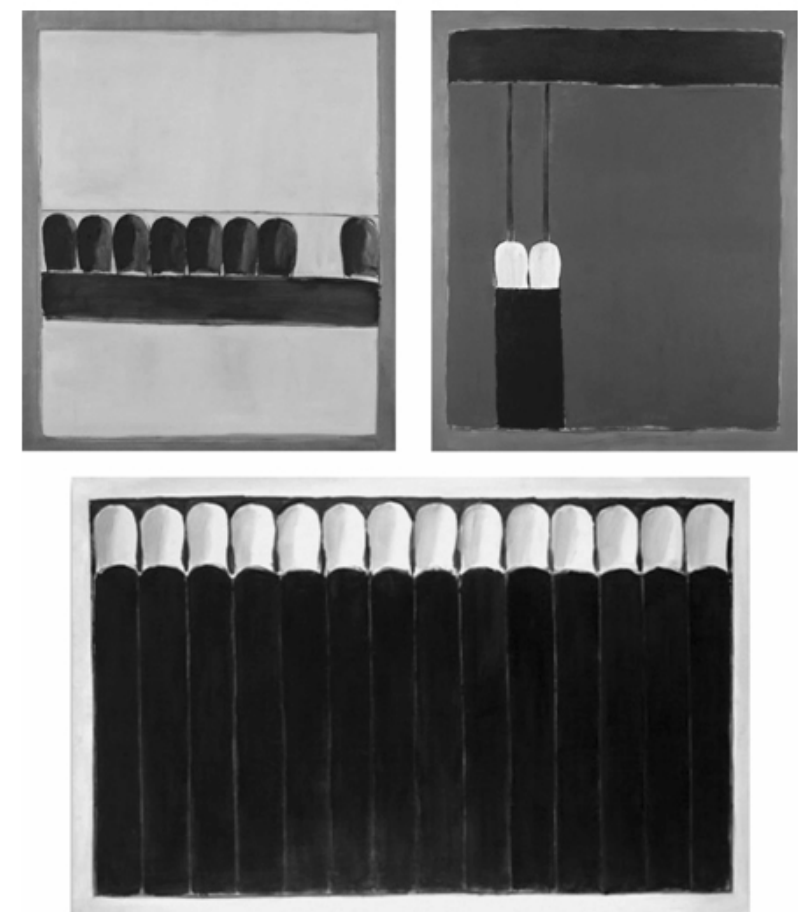

Figura 2A. José Guerrero, Solitarios (1971). Figura 2B. José Guerrero, Solitarios (1972). Figura 2 C. José Guerrero, Intervalos negros (1971), procedencia: Centro José Guerrero.

El espectrofotómetro de reflectancia usado cuenta con un registro de longitudes de onda establecido entre 360-740 nanómetros (nm), lo que está considerado como similar a la percepción de un observador medio, con un intervalo de longitud de onda de 10 $\mathrm{nm}$ y un observador patrón de $2 / 10^{\circ}$ (de modo que los estímulos que activarían su retina estarían situados en un ángulo visual entre $2^{\circ}$ y $10^{\circ}$ (CIE 1931/2 $2^{\circ}$, CIE 1964/10 $)$. También establece un observador estándar equiparado a la visión humana y el iluminante normalizado D65, igualado a la luz intensa de un día luminoso en Europa septentrional. Además, este espectrofotómetro mide la cantidad proporcional de luz reflejada por la superficie de una muestra dada, interpretando las longitudes de onda para producir un espectro de reflectancia (entendiendo la reflectancia lumínica de una superficie como la propiedad de ésta para reflejar la fracción de la luz que no absorbe tras ser iluminada, siendo medida en \%). De esta manera, se pueden determinar los valores triestímulos CIE 
XYZ (rojo, verde, azul) para las muestras tomadas, bajo el iluminante D65.

Antes de las mediciones, el espectrofotómetro debe ser calibrado según un color blanco estándar. Realizadas las mediciones, se procede a la descarga de datos mediante la aplicación informática Color Data Software CM-S100w SpectraMagic TM NX. A cada muestra de color le es asignada una unidad numérica, que hace referencia a sus propias características, esto permite que los resultados puedan quedar reflejados en diferentes imágenes gráficas según las características del color: reflectancia porcentual, luminosidad, parámetros del color y diferencia de croma (respecto a una muestra patrón dada).

En todo momento, se ha trabajado con una metodología consistente en técnicas de análisis no destructivas. Los datos han sido tomados directamente de las superficies originales de las pinturas, sin provocarles alteraciones y sin extraer muestras de materiales.

\subsection{Elementos auxiliares}

En los diferentes ensayos, hechos sobre las obras pictóricas de José Guerrero, han resultado necesarios una serie de elementos auxiliares, que han posibilitado la realización de los mismos de manera ordenada y clasificadora.

En primer lugar, se han realizado mapas de trabajo de los cuadros a estudiar. Para confeccionarlos se ha partiendo de las imágenes de las pinturas originales, pero con menor intensidad de color y luminosidad, y se les han superpuesto redes cuadriculadas con divisiones equidistantes. Estas medidas han sido proporcionales a las que se han establecido en las pinturas reales en el momento de tomar las muestras, de manera que cada división de la cuadrícula del mapa ha correspondido a una representativa de 10 $\mathrm{cm}$ de lado en las obras originales. En el cruce de líneas han quedado fijados los puntos donde se han hecho las mediciones. Los mapas se imprimieron en papel, resultando ser de diversas dimensiones, pues cada uno de ellos ha sido proporcional al cuadro representado, teniendo diferente número de registros.

Las mediciones se realizaron en las pinturas originales colocando cintas métricas de tela (confeccionadas a propósito para no dañar la superficie pictórica), transportando el diseño de cada cuadrícula sobre su obra original, siendo las distancias de los espacios de las redes cuadriculadas de $10 \mathrm{~cm}$ reales, y usando el espectrofotómetro. Descargados los registros obtenidos en el ordenador, se confeccionaron los gráficos respectivos, según divisiones de unidades colorimétricas, que establecieron las escalas numéricas de los datos de color.

\section{Resultados y discusión}

En la etapa creativa de José Guerrero conocida como Fosforescencias (1970-1973), a la que pertenecen las tres pinturas estudiadas en este trabajo, el artista tuvo como referente figurativo grupos y cajas de cerillas de carterilla (Guerrero, 2003). Además, se advierte que en este estudio los colores de los gráficos de las Figs 3,4 y 5 se ajustan a valores numéricos cromáticos. Es por esto que no deben buscarse similitudes entre los tonos de dichos gráficos y los de las pinturas a las que representan. 


\subsection{Solitarios (1972)}

El cuadro original Solitarios (1972) (Fig. 3) está pintado en colores verdes, grises, negros y blancos. Haciendo un análisis simbólico, el color verde es el que más se relaciona con la naturaleza, el crecimiento, la armonía, la fertilidad, la frescura, la calma, la esperanza, etc. El verde oscuro unido al negro es el principio de la muerte, la negación de la vida, la pena. En esta obra, los grises son un puente, una bisagra entre los tonos oscuros y el blanco, puro y regenerador, que aporta interés y motivación a la mirada por sus características de intensa luminosidad.

La imagen de la Fig. 3 presenta los resultados correspondientes a las diferencias de croma entre áreas del cuadro Solitarios (1972). Las mediciones se tomaron en 2009. Los valores cromáticos han quedado establecidos entre 0 y 45 unidades de croma, tras hacer constar los máximos y mínimos registrados, diferenciándose 5 áreas distintas. Se hicieron un total de 437 mediciones sobre la superficie total del cuadro $(216,5 \times 183 \mathrm{~cm})$. En la Fig. 3 aparece la pintura original en la parte superior, en la zona baja está su gráfico correspondiente y a la derecha de éste está situada la escala de color utilizada.

El gráfico (Fig. 3) define áreas de igualdad de croma, con una distribución similar a las manchas definidas en la pintura original; si bien se observan entre ambos diferencias importantes de difícil apreciación sólo con la observación visual.

En la representación colorimétrica (Fig. 3) se exponen con un mismo croma las zonas que en la pintura aparecen como grandes manchas de color (área e). Tanto el perímetro como los grandes planos centrales han sido representados formando parte del área e. Los colores más oscuros de la pintura están definidos en las áreas c, con la característica de que éstas quedan bordeadas por el área $\mathrm{d}$. Este área d se distribuye en el cuadro en las zonas limítrofes de los colores dominantes, que dan origen a las formas del dibujo.

Las manchas pictóricas más claras están representadas en el gráfico (Fig. 3) con el color del área $\mathrm{b}$ y en el interior de ésta se observa un valor numérico de croma definido con el área a, que proviene del valor más sombrío de esta zona.

La utilización de diferentes colores en la pintura está representada en el gráfico por la variabilidad de sus valores cromáticos, que en esta obra es reducida. José Guerrero trabajó el cuadro con una paleta de color limitada a los tonos fríos, en una etapa artística en la que usó como referente imágenes figurativas sintetizadas (cerillas). Esto le sirvió de motivo expresivo, manteniendo, a su vez, las mismas características técnicas de su etapa abstracta anterior, como el interés por los espacios y las formas de la composición, la libertad en el uso del color y la distribución de manchas pictóricas con separación entre ellas, dando lugar a que "respirasen" dentro del cuadro. En el gráfico se aprecia claramente la importancia que el pintor dio a las transiciones entre los límites de diferentes manchas de color, que les proporcionan vibraciones visuales, realzan estas zonas y las integran en la obra. Se trata de una composición en la que destaca la importancia dada a la distribución del plano pictórico, donde el cuadrante inferior izquierdo soporta el peso visual de la parte superior, formada por una gran mancha horizontal de tono oscuro, que se encuentra cercana a su margen. 


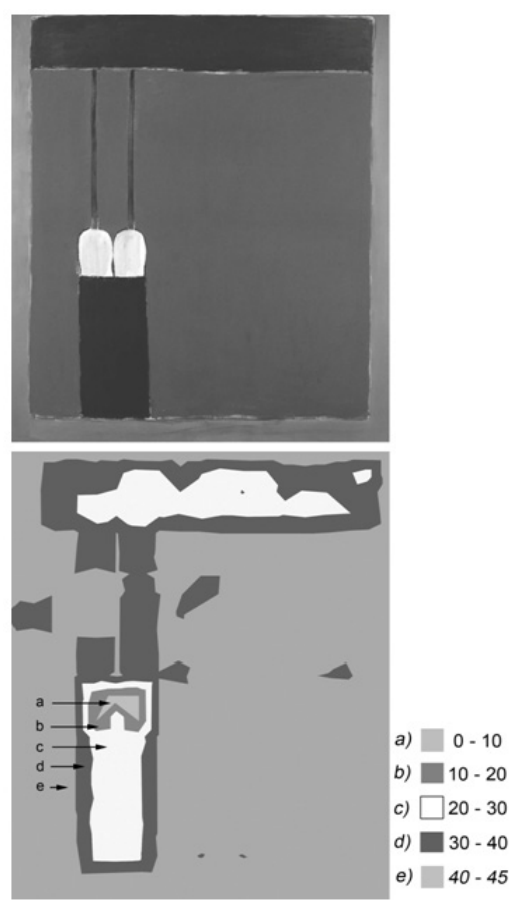

Figura 3. José Guerrero, Solitarios (1972). La imagen de la parte superior corresponde al cuadro original que ha sido analizado colorimétricamente, procedencia: Centro José Guerrero. La imagen inferior representa el gráfico de isocromas de la obra, donde se observan diferentes áreas de igualdad de croma, según la ecuación

CMC (1: c) (D65). La escala gráfica (colores y letras) muestra diferencias de diez unidades de croma, autoría: M. C. Bellido Márquez.

\subsection{Solitarios, 1971}

El cuadro Solitarios (1971) (Fig.4) está pintado en colores amarillos, grises, negros y blancos, con contrastes muy fuertes. Estas matizaciones de color se pueden relacionar con las connotaciones que posee el color amarillo, que están asociadas a la luminosidad, la claridad, la calidez, la divinidad, los rayos solares, el día, la primavera, la alegría, el valor del oro, el vino blanco, el fuego, etc.; también este color tiene resonancias negativas como la envidia, la angustia, la mala suerte, la política, la economía, la prensa, la fiebre y otros males humanos. De esta variedad de iconos simbólicos y culturales merece la pena recordar que, por encima de todos ellos, el amarillo se conoce universalmente como referente de la luz. Por el contrario, las sugestiones simbólicas y psicológicas del color negro son la opacidad, la oscuridad, la tristeza, la sobriedad, la nocturnidad, la suciedad o la maldad, enfrentadas a otras connotaciones positivas como la distinción, la elegancia, la rectitud, la seriedad, la autoridad, etc. El blanco y el gris de esta obra forman el soporte de las transiciones de tonos, que son muy importantes en este trabajo.

La Fig. 4 presenta los resultados correspondientes al estudio de color del cuadro Solitarios (1971). Las mediciones se tomaron en 2009. En la parte superior de la imagen está representada la obra original, bajo ésta ha sido colocado el gráfico, que expone 
las diferencias de croma encontradas entre áreas, a su derecha se encuentra la escala de unidades de color utilizada. Los valores cromáticos han quedado establecidos entre 0 y 90 unidades de croma, tras hacer constar los máximos y mínimos registrados, diferenciándose 6 áreas distintas. Se tomaron un total de 437 mediciones sobre la superficie total del cuadro $(216,5 \times 183 \mathrm{~cm})$.

Se observa (Fig. 4) que las líneas y áreas de igualdad de croma se sitúan en el gráfico de forma equivalente a los colores y manchas de la obra pictórica. La representación gráfica de isocromas muestra una distribución espacial muy parecida entre las áreas de color de la pintura y las del gráfico, con muchos puntos de similitud, aunque se observan diferencias importantes que merecen ser subrayadas, pues en ellas radica la mayor importancia del estudio.

En la pintura destacan dos grandes manchas situadas en la parte superior e inferior del cuadro, que están representadas en el gráfico con el área f (Fig. 4). Las manchas centrales más oscuras se definen como área $\mathrm{b}$ y las que forman el contorno, a modo de marco, se muestran en el diagrama como área a. En los bordes de las grandes áreas f, que corresponden a manchas amarillas, se observa la importante variabilidad cromática que existe, quedando definida por nuevas áreas. Esto corrobora la no uniformidad del plano $\mathrm{f}$ y su riqueza de matices, aunque es difícilmente apreciable a la vista humana en la pintura original o en su representación fotográfica.

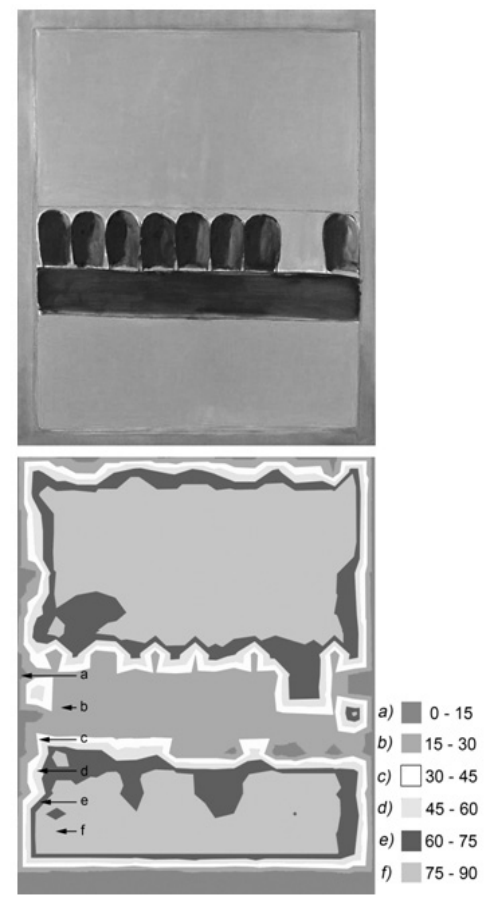

Figura 4. José Guerrero, Solitarios (1971). La imagen de la parte superior corresponde al cuadro original que ha sido analizado colorimétricamente, procedencia: Centro José Guerrero. La imagen inferior corresponde al gráfico de isocromas de la obra, donde se observan diferentes áreas de igualdad de croma, según la ecuación CMC (l: c) (D65). La escala gráfica (colores y letras) muestra diferencias de quince unidades de croma, autoría: M. C. Bellido Márquez. 
Se distingue en el gráfico (Fig. 4) la similitud de croma existen entre las zonas de los bordes del cuadro y las machas centrales más oscuras, pues están igualmente representadas con áreas $\mathrm{b}$. Se forma así una zona de color perimetral que unifica los tonos y les da unidad. Se comprueba la gran riqueza de matices que posee el cuadro entre los colores colindantes de las diferentes manchas pictóricas, ofreciendo degradaciones de unos a otros (áreas b, c, d y e). Son evidentes las gradaciones de áreas desde los bordes del cuadro hacia el interior. Todo ello permite comprobar que la cromaticidad de los tonos centrales es igual, en general, al degradado que se produce en las zonas cercanas al perímetro de la obra, si bien, visualmente este detalle es difícilmente observable.

Las matizaciones de color que presenta el cuadro se pueden relacionar con las obras de pintores que trabajaron los campos de color (color-fields paintings), dentro del Expresionismo Abstracto americano, interesados por la riqueza de matices en las transiciones de tonos, como es el caso del contemporáneo y amigo de José Guerrero, el artista Mark Rothko. Esta característica de trabajo la desarrolló Guerrero ya en cuadros precedentes a los estudiados aquí, pertenecientes a su etapa expresionista abstracta, influido por el movimiento americano mientras vivía en Nueva York y se relacionaba con importantes artistas unidos a este movimiento, como Franz kline, Mark Rothko, Clyfford Still y Barnett Newman. Como expresionista, Guerrero realizó obras que destacan por su cromatismo y disposición de las manchas de color, características que compartió con el estilo de la action painting y que mantuvo en sucesivos trabajos (como en esta pintura), mezclándolas con nuevas forma de trabajo.

\subsection{Intervalos negros (1971)}

La obra original Intervalos negros (1971) (Fig.5) está pintada en colores blancos, negros y grises. Para Guerrero, el negro fue el color por excelencia, el color de los colores, que estuvo siempre presente en su obra, como patrimonio espiritual de su memoria remota, que se manifestaba en sus recuerdos personales, siendo símbolo del luto o de la tormenta, en muchas ocasiones. Es un color que formó parte de su paleta de trabajo desde la creación de sus primeras obras, como sombra constante que acompaña la irradiante luz de la vida. Por otro lado y debido a la simbología social, el color gris se asocia a la tristeza, a la vida anodina, al mal tiempo, a la tormenta, a lo sombrío o lo poco lúcido, pero también se relaciona con la inteligencia, dada a su afinidad pigmentaria con la materia gris del celebro. Por el contrario, el blanco se corresponde con la claridad, la evidencia, la limpieza, la virginidad, la pureza, la purificación, la inocencia, la paz, etc.

La imagen de la Fig. 5 presenta los resultados correspondientes a las diferencias de croma entre áreas del cuadro Intervalos negros (1971). En la parte superior se encuentra representada la pintura original. Bajo ella está situado el gráfico de isocromas y a la derecha de éste se puede ver la escala de unidades de color utilizada. Las mediciones se hicieron en 2009. Los valores cromáticos han quedado establecidos entre 0 y 54 unidades de croma, tras constar los máximos y mínimos registrados, diferenciándose 4 áreas distintas de color. Se registraron un total de 551 muestras sobre la superficie total del cuadro $(180 \times 278 \mathrm{~cm})$.

A primera vista, se observa en el gráfico (Fig. 5) que las líneas y áreas de igualdad de croma se distribuyen de modo parecido a los colores y manchas pictóricas del cuadro, 
pero destacando diferencias importantes entre la imagen pictórica y la gráfica.

Se observa (Fig. 5) que las manchas de colores grises claros de la pintura están representadas gráficamente por el color del área a. Es importante destacar que los límites entre las manchas pictóricas quedan representados en el gráfico con un único valor cromático que las bordeas, que está formado por el área d.

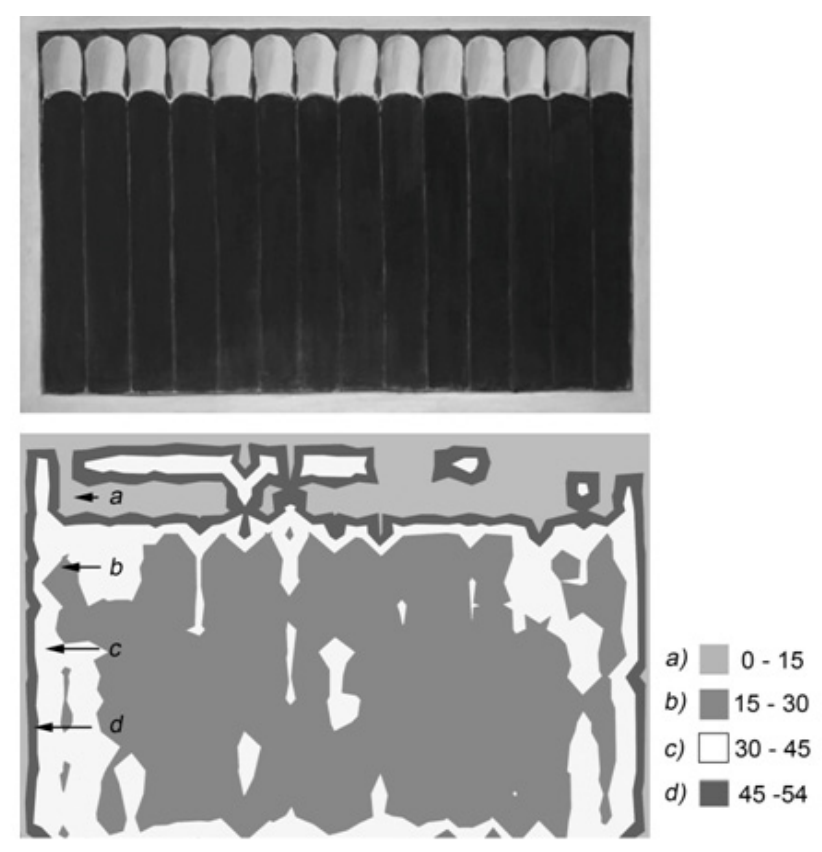

Figura 5. José Guerrero, Intervalos negros (1971). La imagen de la parte superior representa el cuadro original que ha sido analizado colorimétricamente, procedencia: Centro José Guerrero. La imagen inferior corresponde al gráfico de isocromas de la obra, donde se observan diferentes áreas de igualdad de croma, según la ecuación CMC (1: c) (D65). La escala gráfica (colores y letras) muestra diferencias de quince unidades de croma, autoría: M. C. Bellido Márquez.

La gran mancha oscura que ocupa la mayoría de la superficie de la pintura, se representa en el gráfico (Fig. 5) con el área b. Junto a ella se encuentran matices cromáticos de valores similares entre sí, representados con áreas c, que muestra que el área $\mathrm{b}$ no es monocromática, sino que presenta variedad de croma dentro de ella. El límite del área $\mathrm{c}$ se representa con el área d, igual que las transiciones de las manchas superiores y las del contorno del cuadro.

José Guerrero usó una paleta de colores reducidos para realizar esta pintura. Sus cromas han quedado estabecidos gráficamente (Fig.5) en cuatro valores diferenciados. Se trata de un ejercicio de síntesis en el que han sido utilizados colores bastante acromáticos, cercanos al gris, negro y blanco neutros. Como en otras obras del artista, los límites entre diferentes manchas de color poseen definiciones cromáticas propias, originadas por las mezclas y degradaciones entre diferentes pigmentos. Esto se ve enriquecido con 
colores provenientes de la capa de preparación, del soporte o de capas base, pues un examen visual de la obra permite observar pequeños espacios libres de pintura que dejan ver la tela y tonos grisáceos, difíciles de apreciar en la imagen fotográfica, pero bien identificados en este estudio.

El tema representado por el artista en esta obra (Fig. 5) son las cerillas alineadas. Ha sido realizado de manera austera en cuanto a combinación de colores y composición. El dibujo se define por la colocación de formas en sentido vertical, el cual genera una secuencia horizontal, marcada por su aparente falta de cromaticidad, que en realidad es algo más rica de lo que se aprecia a simple vista, a tenor de lo que se observa en las transiciones de manchas, aunque entre los colores se registran, principalmente, fuertes contrastes de luminosidad, más que de tonos. Es la intensa luminosidad del color blanco y gris claro, frente a la escasa del negro, lo que permite diferenciar las formas de la composición pictórica. Entre los márgenes de estas formas, definidas por sus colores, destacan zonas de transición con valores visuales de color que enriquecen la imagen.

\section{Conclusiones}

En primer lugar, se piensa que el logro político y social que ha significado mantener la colección Guerrero en el Centro José Guerrero (Granada) es una victoria para la difusión del arte. De igual manera, el propósito de realizar este estudio científico sobre el color de un grupo importante de obras que la componen ha supuesto una enorme satisfacción, al ser acometido en tiempos donde se temía por la retirada de los cuadros de la ciudad de Granada y habiéndolo podido concluir en estas circunstancias, obteniendo resultados de gran interés. Ambos logros permitirán seguir avanzando en la investigación científica y artística de esta colección, en favor de una sociedad que, cada vez más, valora el arte como definición de identidad y lenguaje de comunicación universal, que debe subsistir incluso en difíciles circunstancias.

La motivación de Guerrero por la expresión máxima del color (Navarro, 2011), al que atribuía recuerdos de su infancia y juventud en su tierra natal de Granada y los paisajes que en ella observaba, quedan patentes en sus obras (Ortuño, 1981). La expansión del color y la libre contaminación de tonos fueron utilizadas por el artista como efectos positivos, que debían producirse durante la ejecución de las obras mediante transiciones tonales, que conseguía aplicando diferentes colores directamente sobre la superficie del lienzo en húmedo sobre húmedo y después frotando por encima, obtenido como resultado: mezclas, difuminados o superposiciones. Según él mismo describió: "Ahí nacen esas fronteras, que yo digo, en los bordes de la masa de color. La línea la pongo a veces como línea y otras como transición entre dos tonos" (Ortuño, 1981, p. 147). Esas fronteras generan tonos indefinidos que han sido medidos colorimétricamente y ahora se conocen sus parámetros con exactitud, aunque visualmente sean de difícil definición.

Las mediciones del color, hechas mediante espectrofotometría, verifican la riqueza plástica y técnica del artista José Guerrero, debido a la variabilidad cromática de sus obras y a la creatividad de su técnica. Las aparentes manchas de coloraciones planas que presentan sus cuadros son ricas en matices cromáticos, producidos por la aplicación de colores (muchos de ellos mezclados sobre la propia superficie del lienzo) y la superposición de capas de pintura; todo ello con diferentes densidades, que hacen que un mismo color ofrezca tonos distintos. 
En la valoración del color de las obras de Guerrero destaca el especial interés que tienen los límites entre las distintas manchas de coloración de las obras estudiadas. Se observan con claridad las transiciones progresivas de un color a otro, debido a degradados, a contaminaciones de colores, a la aparición de manchas pictóricas de estratos inferiores o a espacios sin pintar, donde se ve el material del soporte original o la capa de imprimación. En estas áreas abunda la cromaticidad, según ha quedado reflejado en los gráficos obtenidos en este estudio.

El resultado global de la técnica de trabajo de José Guerrero es una obra pictórica vibrante, de contrastada luminosidad, que evidencia su relación con el movimiento expresionista abstracto americano y que atrae la atención del espectador, debido a los contrastes visuales que presentan sus obras. Éstas se caracterizan también por la presencia de grandes espacios de colores de apariencia casi plana, con motivos abstractos o figurativos que carecen de detalles, con gran dominio del lenguaje plástico. Son obras hechas en formatos medios o grandes, que hacen que frente a ellos el espectador se vea inmerso en planos de colores que activan su sentido visual y le provocan diversas sensaciones, debido a la facilidad que tiene la vista humana para recoger información sobre los colores, los cuales son interpretados por el cerebro como mensajes (Spillmann $\&$ Werner, 1990) y analizados por éste bajo prismas emocionales, culturales, religiosos, educacionales, etc.

José Guerrero conoció la comunicación sinestésica del color, capaz de transmitir ideas emocionales, asociadas a recuerdos de vivencias sensoriales. Experimentó con distintas combinaciones e introdujo en sus obras interesantes zonas de transición entre coloridos diferentes, zonas donde han sido detectadas manchas subliminales de color, no percibidas con facilidad por el ojo humano (Gegenfurtner \& Sharpe, 1999), que bien determinadas mediante el análisis colorimétrico, permiten verificar la hipótesis de la riqueza visual y cromática que el artista puso ante nuestros ojos para el deleite de la mirada y el disfrute emocional del observador, según los estudios realizados por la percepción humana del color (Schwartz, 1999) y los efectos psicológicos que la visión del color producen en el espectador (Heller, 2004). Por ello, las relaciones connotativas de los colores que utilizó el autor en estas obras se pueden analizar desde un sentido simbólico, por su importancia como vehículo de comunicación expresiva, de ahí la relevancia de mantener de manera óptima sus tonalidades y evitar su alteración.

En el caso concreto de las pinturas de J. Guerrero estudiadas en este trabajo, no hay que olvidar las composiciones artísticas geométricas, que dividen los planos pictóricos en grandes zonas de colores, enmarcadas por perímetros que determinan los bordes de los cuadros. Los temas son sencillos, como lo son las cerillas presentada en cajas de carterilla, que le sirvieron de inspiración para la realización de estas obras, en las que la sencillez del tema y la forma evidencian el interés de Guerrero por el profundo análisis de la composición gráfica y tonal, dando lugar a que un motivo cotidiano se convierta en el origen de una obra única, en la que la redimensión, a mayor escala del objeto original, da lugar a la descontextualización del mismo y a tener de él una interpretación abstracta, lo que se suma a la importancia que tiene del color como elemento compositivo y vía de comunicación.

Por último, los datos de color obtenidos han permitido la catalogación colorimétrica de las obras estudiadas mediante el registro de sus parámetros, según tablas de 
mediciones numéricas obtenidas de los colores, que han sido conservadas. Con ellas se podrá detectar en el futuro si se producen variaciones de color y determinar su nivel. Igualmente, ayudarán a restaurar las obras adecuadamente. Esta información también resultará útil en la reproducción de las pinturas para su uso en publicidad o difusión.

\section{Referencias}

Arias, J. (2009, viernes, 24 de abril). Revuelta popular en defensa de la continuidad del Centro Guerrero. Granada Hoy, pág. 62.

Bellido, M. C. y Durán, J. A. (2007). La conservación de la colección del Centro José Guerrero de Granada. En Museo Nacional Centro de Arte Reina Sofía, Ministerio de Cultura (Ed.), Conservación de arte contemporáneo: $8^{a}$ Jornada, febrero 2007 (75-83). Internacional Institute of Conservation, Grupo Español. Madrid: Museo Nacional Centro de Arte Reina Sofía, Ministerio de Cultura.

Bellido, M. C.; Durán, J. A. y Peralbo, R. (2008). Evaluación del color en la obra de José Guerrero mediante herramientas científicas de determinación colorimétrica. EnMuseo Nacional Centro de Arte Reina Sofía, Ministerio de Cultura (Ed.), Conservación de arte contemporáneo: $9^{a}$ Jornada, febrero 2008 (47-56). Internacional Institute of Conservation, Grupo Español. Museo Nacional Centro de Arte Reina Sofía, Ministerio de Cultura (47-56).

Bellido Márquez, M. C. (2010). Estudio conservacional y análisis material de obras de arte contemporáneo. Un caso experimental: colección del Centro José Guerrero. (Tesis Doctoral, inédita). Universidad de Granada, Granada (España).

Centro José Guerrero (2012). La colección del Centro. Centro José Guerrero. Recuperado de http://www.centroguerrero.org/index.php/del_Centro/19/0/?\&L=0 [Consulta 10/12/2012].

Europa Press (2011, 1 de diciembre). El Centro Guerrero dedicará 2012 a la obra de Rivera y a la música. La familia y las instituciones trabajan para definir en un futuro las instalaciones que albergará la obra de José Guerrero. Europa Press. Recuperado de http://www.europapress.es/andalucia/cultura-00621/noticia-centro-guerrerodedicara-2012-obra-manuel-rivera-musica-espacio-museo-20111201133135.html [Consulta 9/11/2012].

Idealtv.es (2009). Pancarta Guerrero en la Torre de la Vela por el Centro. Plataforma Por el Guerrero. Recuperado dehttp://www.ideal.es/videos/gente/sociedad/692371621001pancarta-torre-vela-centro-guerrero.html [Consulta 27/04/2009].

Gallastegui, I. (2010, 18 de septiembre). La familia Guerrero renuncia a constituir una Fundación con la Diputación. El Ideal, pág. 38.

Gegenfurtner, K. R. \& Sharpe, L. T. (1999). Color vision: from genes to perception. Cambridge: Cambridge University Press.

Guerrero, J. (2000). Catálogo de la exposición José Guerrero: La colección del Centro. Granada: Diputación de Granada.

Guerrero, J. (2003). Catálogo de la exposición Fosforescencias y otros objetos cotidianos en la pintura de José Guerrero (julio-octubre de 2003). Granada: Diputación Provincial de Granada. 
Heller, E. (2004). Psicología del color: cómo actúan los colores sobre los sentimientos y la razón. Chamorro Mielke, J. (traduc.). Barcelona: Gustavo Gili.

Millán, M. (2009, 17 de noviembre). Entrevista a Lisa Guerrero. Laopiniónde granada. es. Recuperado de http://www.laopiniondegranada.es huella/2009/11/17/

duro / -poner-enfasis-vida-padre-interesa-vivir/126426.html [Consulta 17/11/2009].

Navarro, M. (2011). Colours. Una biografía del pintor José Guerrero. [Película documental]. La Nave de Tharsis en colaboración con Canal Sur Televisión (prod.). Estreno en Sevilla, 11 de noviembre 2011. Por el Centro J. Guerrero (2012). Recuperado de http://www.porelcentroguerrero.com/ [Consulta 7/12/2012].

Ortuño, P. (1981). Conversación con José Guerrero. En Dirección General de Bellas Artes, Archivos y Bibliotecas, España (Ed). José Guerrero. Catálogo de exposición (diciembre 1980-enero-1981) (85-151). Madrid: Ministerio de Cultura. Dirección General de Bellas Artes, Archivos y Bibliotecas.

Romero, Y. \& Vallejo, I. (2007). José Guerrero: Catálogo Razonado, Vol. I. Baena, Fco. (col.). Granada: Centro José Guerrero.

Romero, Y. \& Vallejo, I. (2008). José Guerrero: Catálogo Razonado, Vol. II. Baena, Fco. (col.). Granada: Centro José Guerrero, Telefónica D. L.

Schwartz, S. H. (1999). Visual perception. Stamford: Appleton and Lange.

Spillmann, L. \& Werner, J. S. (1990). Visual perception: the neurophysiological foundations. San Diego: Academic Press. 
